\title{
Fragments sur le travail intellectuel
}

\section{Andrea Cavazzini}

URL : http://journals.openedition.org/grm/754

DOI : $10.4000 / \mathrm{grm} .754$

ISSN : $1775-3902$

\section{Éditeur}

Groupe de Recherches Matérialistes

\section{Référence électronique}

Andrea Cavazzini, «Fragments sur le travail intellectuel », Cahiers du GRM [En ligne], 9 | 2016, mis en ligne le 30 juin 2016, consulté le 01 mai 2019. URL : http://journals.openedition.org/grm/754 ; DOI $10.4000 / \mathrm{grm} .754$

Ce document a été généré automatiquement le 1 mai 2019.

(c) GRM - Association 


\title{
Fragments sur le travail intellectuel
}

\author{
Andrea Cavazzini
}

I

1 L'une des contributions fondamentales de l'opéraïsme consiste à avoir essayé de penser jusqu'au bout le travail comme une réalité structurellement divisée en deux. Comme le rappelle Luciano Ferrari Bravo :

La duplicité du travail, qui constitue justement la problématique de Marx, s'avère, dès qu'elle est envisagée dans des termes historiquement déterminés, qualifiée par la forme capitalistique qu'elle a prise. Elle se présente donc comme une duplicité entre le travail mort et le travail vivant. Il est possible de rattacher en dernière instance à ce couple fondamental toute la série de dualismes qui se manifestent dans le mode spécifiquement capitaliste de production: travail et force-travail, processus de travail et processus de valorisation, travail concret et travail abstrait etc. L'opposition - dialectique, irréductible à la contrariété - entre travail vivant et travail mort se prolonge dans tous les autres couples. Prenons par exemple le couple force-travail/travail. La force-travail est, d'une part, du travail mort, puisqu'elle équivaut à la quantité de valeur déjà produite qui est achetée sur le marché en tant que salaire ; mais, d'autre part, la force-travail présente un profil historico-social, déterminé en dernière instance par la subjectivité ouvrière vivante. Symétriquement, le travail - l'autre terme du couple - est la source de la valeur, une source parmi d'autres du point de vue de la richesse réelle, mais la seule et unique du point de vue de l'accumulation capitaliste: néanmoins, c'est précisément à cause de cette vertu «créatrice » que le travail représente le plus grand malheur pour l'ouvrier' ${ }^{1}$.

2 Cette idée d'une duplicité originaire du travail - que la forme capitaliste de l'activité humaine incarne dans une séquence historique déterminée - permet peut-être de penser le travail intellectuel par-delà les exercices sociologiques sur les «intellectuels » ou la querelle interminable et vaine du rapport entre « théorie » et praxis politique. 
3 Sergio Bologna a caractérisé la duplicité spécifique du travail dit « intellectuel » dans les termes d'une scission - qui ne se présente jamais en tant que telle dans la réalité concrète - entre, d'une part, l'activité créatrice de connaissance et/ou de culture, et, d'autre part, la production de compétences ayant une valeur marchand ou institutionnel :

Le travail intellectuel rétribué est un échange marchand portant sur des compétences ; il n'est pas un travail de connaissance, si bien que, après avoir fourni des services-compétences nous ne nous sentons guère enrichis en ce qui concerne nos connaissances. Nous avons augmenté notre savoir-faire, qui est une chose bien différente : nous sommes mieux outillés pour fournir un service analogue avec un effort moindre, tout comme l'ouvrier, après avoir répété cent fois le même geste, sait comment accomplir celui-ci tout en lui arrachant le temps pour une cigarette sans réduire les outputs produits. Mais cette situation qui est typique du skill, du savoir-faire, n'appartient pas à la sphère du travail de connaissance, lequel reste par sa propre nature un travail extérieur au marché, disjoint tant d'un produit spécifique que du "produire " en tant que tel - un travail lié à l'invention, à l'innovation, à la rupture des schémas habituels, à la libération. Connaissance et liberté sont deux termes indissociables, qu'on peut exprimer aussi avec le terme de "liberté de pensée ", qui est quelque chose qui ouvre sur l'infini. Le skill est au contraire toujours limité à quelque chose de fini et de contraignant, comme toutes les prestations inscrites dans l'échange marchand: il relève d'une liberté limitée, d'un rapport instrumental et de dépendance. En outre, la sphère de la connaissance n'est jamais spécialisée, tandis que la compétence doit nécessairement se spécialiser. Ainsi, la connaissance innerve la transformation sociale ; elle est par nature incompatible avec la marchandisation, ne s'inscrit pas dans le cycle marxien argent-marchandise-argent, c'est un produit de la gratuité. Parler d'un «travail de connaissance " est donc partiellement trompeur : le terme de "travail » devrait être toujours associé à l'idée du travail en tant que marchandise échangeable avec de l'argent ${ }^{2}$.

Dans les activités « intellectuelles » il y a donc une composante, un moment inéliminable, qui est irréductible à l'échange marchand et partant à la valorisation capitaliste. Un moment qui n'a pas de valeur pour le capital et qui n'est donc pas reconnaissable en tant que travail productif au sens capitaliste.

\section{III}

Ce moment est irréductible parce qu'il semble impossible de le soumettre à des normes extérieures et préétablies: l'« infinitude» de l'activité intellectuelle «créatrice» ne consiste pas dans une illimitation quantitative - le brassage de masses de données gigantesques ne contient rien d'irréductible à la norme de la valorisation - mais dans l'excès que représente une activité susceptible de se donner elle-même ses propres fins, sa propre forme, et de faire ainsi de sa forme, qui coïncide à la limite avec cette activité elle-même, sa propre fin immanente. Cette finalité autonome et interne est indissociable d'une position subjective singulière qui caractérise les porteurs de ces activités :

Dans le travail intellectuel l'aliénation n'est jamais totale - l'aliénation qui donnait à l'ouvrier-masse le sentiment d'une extranéité absolue par rapport à son produit et qui lui ouvrait l'esprit, le rendait prêt pour le conflit (...). Le travail intellectuel ne parvient pas à se détacher entièrement de son produit, il n'atteint jamais cette aliénation totale qui lui permet de comprendre le fonctionnement objectif du 
monde. Quelque chose de lui, un moment intime de lui-même, rentre dans le produit, si bien qu'il devient difficile de porter sur celui-ci un regard détaché ${ }^{3}$.

Le travailleur intellectuel n'est jamais un simple porteur contingent de " compétences » objectivées - il est aussi un tel porteur, ce qui fait de lui de la force-travail échangeable ; mais son aliénation incomplète l'expose constamment à une division qui le fait virtuellement vivre sur une autre scène, celle du « royaume des fins » et de l'expression libre de soi. C'est ce que rappelle Jean-Claude Milner :

Ce qui caractérise l'intellectuel, c'est évidemment la nature « intellectuelle » de son objet. Nul, malheureusement, n'a jamais bien su ce que cela voulait dire; on peut admettre pourtant que quelque chose de l'ordre de l'universel y est impliqué (...). Mais ce qui caractérise l'intellectuel, c'est encore autre chose : l'intérêt ou plutôt la passion qu'il nourrit pour ce qu'il a élu comme son objet (...). L'intellectuel, en bref, est quelqu'un qui s'intéresse à ce qu'il fait et qui, en tant du moins qu'il fonctionne comme intellectuel, fait ce qui l'intéresse ${ }^{4}$.

7 Cet intérêt passionné qui définit subjectivement l'intellectuel ne distingue pas des catégories socio-professionnelles définies - tandis que l'élitisme républicain défendu par Milner voudrait reconnaître une distinction «objective» entre, d'une part, les intellectuels « authentiques » et, d'autre part, les journalistes, fonctionnaires, opérateurs des médias, etc.

En réalité, la division affecte tous les porteurs des activités intellectuelles et toutes les couches socio-professionnelles en lesquelles ceux-ci se distribuent - car cette division consiste précisément dans l'existence, au sein de la composition du travail intellectuel, d'un moment qui n'est jamais « compté » dans les équivalences de l'échange marchand et dans l'ordre des tâches sociales et institutionnelles. Toute activité qu'on peut reconnaître comme appartenant à la sphère intellectuelle contient à la fois une inscription sociale objective qui la rend inévitablement "fonctionnelle » et un moment "vocationnel » d'autodétermination et d'expression. Ainsi, tout travail intellectuel est clivé entre autonomie et hétéronomie, liberté et servitude.

\section{IV}

Cette division entraîne plusieurs conséquences - et contradictions.

10 Premièrement, le travail intellectuel représente, à travers précisément sa relative nonaliénation, une image ou une figure de ce que pourrait être une activité humaine générique non soumise aux impératifs de la valorisation du capital et des structures sociales et institutionnelles dominantes - il nous rappelle qu'une activité non aliénée est concevable comme horizon d'auto-réalisation de l'agir humain, que des rapports non hétéronomes peuvent exister entre l'action, son produit et ses circonstances.

11 Toutefois, cette image de la non-aliénation est elle-même aliénée, puisqu'elle ne se présente que dans la sphère des formes, des langages et des théories et ne devient effective que sur le plan de l'élaboration symbolique du monde. D'où une nouvelle contradiction : la liberté qui se manifeste dans le travail intellectuel fait allusion - per speculum et in aenigmate - à une réappropriation des conditions communes de l'existence qui concerne l'organisation des "destinées générales»; mais elle présente cette réappropriation comme quelque chose qui pourrait se réaliser dans une sphère séparée, toujours-déjà soustraite aux nécessités de l'action politique qui vise ces conditions communes au nom de l'organisation de la vie de tous. L'autonomie, la liberté associées à 
l'activité intellectuelle risquent constamment de devenir des libertés fantasmatiques et "spectrales », destinées à l'autoréférentialité d'une pratique symbolique qui s'engendre et se consume elle-même indéfiniment, sans se confronter à l'épreuve du réel que constitue la transformation des rapports sociaux de production.

Deuxièmement, ce travail qui contient une partie non calculable, non évaluable à l'aune des inscriptions et des mandats sociaux, est toujours susceptible de basculer dans l'inutilité, la superfluité et la désocialisation radicales. Les porteurs d'une activité qui résiste par définition à des finalités externes, et qui n'est jamais effectuable sans un intérêt subjectif investi en elle, sont constamment hantés par la marginalité et l'inutilité sociales de leurs pratiques.

13 Jean-Claude Milner a bien vu que le ressort de l'enracinement social extrêmement puissant des intellectuels tout au long de l'histoire française est précisément cette hantise. Si les intellectuels français ont massivement accepté le mandat social que leur assignait l'école, c'est qu'ils en retiraient « un bénéfice dont ils rient volontiers, mais qui leur est secrètement précieux : un accrochage social. La hantise de la plupart d'entre eux a bien été au cours des siècles qu'on les traitât en parasites " :

C'est parce que l'école leur prête son assise, son inscription sociale et sa respectabilité, que les intellectuels peuvent ne pas se tenir pour d'éternels parasites. Ce qu'on appelle la vie intellectuelle en France est, dans son organisation matérielle, fondée sur ce contrat secret ${ }^{5}$.

14 L'activité intellectuelle est généralement liée à un défaut d'adaptation, à une absence de légitimité et de statut dont les conséquences ne peuvent être assumées subjectivement qu'avec une certaine difficulté. L'autonomie à l'égard des rôles et des tâches relevant de l'ordre social existant s'est toujours payée, dans l'histoire sociale des fonctions intellectuelles, d'une condition d'anomie et de déréalisation: dans le privilège de l'élaboration des fins et des significations coïncident l'élection et la malédiction, le vertige du vide et celui de l'Absolu - aujourd'hui, le snobisme élitiste et la précarité.

\section{V}

Le lien entre, d'une part, l'activité intellectuelle et, d'autre part, la fréquentation d'un « royaume des fins » se voulant au-delà de l'aliénation, a pu faire croire, pendant environ deux siècles, en un rapport privilégié - une harmonie préétablie ou un parallélisme caché - entre les pratiques intellectuelles et les luttes des classes laborieuses contre l'exploitation et pour la réappropriation des fins et du sens de leur existence matérielle. Cette croyance a été exprimée de la manière la plus directe et enthousiaste par Paul Lafargue, qui associait immédiatement la libération des ouvriers du joug du travail salarié à la libération des activités intellectuelles du joug de la production capitaliste :

Le producteur de la société communiste labourera et sèmera à la machine aujourd'hui, filera, tournera le bois ou rabotera l'acier demain et exercera tour à tour les métiers les plus divers au grand profit de sa santé et de son intelligence. Les applications industrielles des sciences mécaniques, chimiques et physiques, qui, accaparées par le Capital, martyrisent le travailleur, dès qu'elles seront propriété commune émanciperont l'homme du travail et lui donneront des loisirs et la liberté (...). L'artiste alors peindra, chantera, dansera, l'écrivain écrira, le musicien composera des opéras, le philosophe bâtira des systèmes, le chimiste analysera les corps non pour gagner de l'argent, pour recevoir un salaire, mais pour mériter des applaudissements, pour conquérir des couronnes de laurier, comme les vainqueurs 
des Jeux Olympiques, mais pour satisfaire leur passion artistique et scientifique, car on ne boit pas un verre de champagne et on n'embrasse pas une femme aimée pour la galerie. L'artiste et le savant pourront alors répéter les enthousiastes paroles de Kepler, ce héros de la science: "L'Electorat de Saxe avec toutes ses richesses ne vaut pas le plaisir que j'ai ressenti en composant le Mysterium Cosmographicum ${ }^{6}$.

Pour Lafargue, la passion de l'intellectuel pour sa pratique créatrice est le modèle de l'épanouissement de l'agir humain par-delà l'aliénation capitaliste. Mais l'autre moment de cette pratique, sa disjonction d'avec des inscriptions sociales vérifiables, rapproche l'activité intellectuelle moins de la perspective du travail vivant libéré que de la condition contemporaine de l'inutilité.

Il existe peut-être un parallélisme entre l'inadaptation persistante du travail intellectuel et l'expulsion actuelle d'individus et populations de tout usage socialement légitime de leur existence. Les rapports capitalistes contemporains engendrent une masse croissante de « gens dont on peut dire (...) qu'ils sont comptés pour rien par le capital, ce qui veut dire qu'au regard du développement structurel du monde, ils ne sont rien, et que donc, en toute rigueur, ils ne devraient pas être là » :

Que veut-il dire qu'ils sont comptés pour rien? Cela veut dire qu'ils ne sont ni consommateurs, ni force de travail (...). Votre identité aux yeux du mouvement dominant du monde d'aujourd'hui est la double identité, structurée par l'argent, de salarié et de consommateur (...). Or [ces gens] ne trouvent nul accès ni à l'un ni à l'autre (...). Du point de vue de la logique générale du monde, de l'impérieuse et satisfaite mondialisation capitaliste, ils sont comme s'ils n'existaient pas?

Selon Pierre-Noël Giraud, les « damnés de la terre » étaient au XIXème et XXème siècles les colonisés et les surexploités; au XXIème, ce sont les hommes inutiles »:

Qui sont les hommes inutiles? Les chômeurs de longue durée dans les pays dits riches, mais aussi les working poors et tous deux qui enchaînent des « petits boulots " précaires (...). Soit plus de la moitié des jeunes dans de nombreux pays. Les paysans sans terre, ou qui ont si peu de terre que, pour survivre, ils l'épuisent. Les habitants des slums mal connectés aux centres des villes, sans services publics de base et soumis aux inondations et aux ouragans, qui ne trouvent pas à survivre dans le secteur informel, ceux pour qui la ville ne fonctionne pas comme pourvoyeuse d'opportunités et qui vivent donc de solidarité familiale et clanique ${ }^{8}$.

Or l'inutilité de ces « inutiles » n'est nullement identique à celle qui frappe la composante non-aliénée du travail intellectuel. Car la condition de l'homme inutile ne relève pas principalement d'une vocation ou d'une finalité non calculables par le capital: au contraire, les hommes inutiles «ne sont pas tout bonnement en dehors de la circulation du capital, ils sont activement produits comme non-travailleurs par cette circulation » :

La totalité capitaliste de la production, en dehors de son besoin de travailleurs, engendre l'« armée de réserve » de ceux qui ne parviennent pas à trouver du travail 9 .

Les hommes inutiles qui peuplent les métropoles, les slums ou les territoires désertifiés n'incarnent pas automatiquement une finalité différente de l'accumulation du capital, mais sont toujours-déjà calculés comme les « grandeurs négatives » de celle-ci, comme de la force-travail excédentaire plutôt que comme une activité irréductible à de la forcetravail.

19 Ainsi, l'inutilité du travail intellectuel est à l'inutilité de cette force-travail négative mondiale ce que l'autonomie de la fonction intellectuelle était aux luttes des « damnés de la terre »: un simulacre, à travers lequel les contradictions qui affectent les destinées générales, et leur dépassement virtuel, sont déplacés dans l'espace d'une représentation - 
quelque chose de différent tant d'une simple mystification que d'une réalisation immédiate.

Mais la représentation et le simulacre sont toujours des réalités, produisent des effets, qu'il faut analyser du point de vue de leurs potentialités immanentes.

\section{VI}

21 Cela est d'autant plus vrai que la composante du travail intellectuel qui est homogène aux critères de la valorisation capitaliste tend désormais inexorablement à se rapprocher de la condition de l'homme inutile en général.

La scission interne au travail intellectuel entre une composante valorisable et un moment «autonome » affecte moins aujourd'hui une élite médiatrice et organisatrice chargée d'administrer les idéologies dominantes qu'une vaste strate d'intellectuels-masse dont les conditions d'existence sont de plus en plus déterminées par la précarisation et la subalternité.

22 La contradiction entre les deux moments du travail intellectuel dans les années 1960 et 1970 pouvait encore être exprimée par ce passage de Sartre :

L'ensemble des intellectuels apparaît comme une diversité d'hommes ayant acquis quelque notoriété par des travaux qui relèvent de l'intelligence (science exacte, science appliquée, médecine, littérature etc.) et qui abusent de cette notoriété pour sortir de leur domaine et critiquer la société et les pouvoirs établis au nom d'une conception globale ${ }^{10}$.

Mais cette définition, qui pourrait être incontestable à l'époque de l'affaire Dreyfus, est devenue anachronique face à la subsomption capitaliste réelle du travail intellectuel. Comme le rappelle Romano Luperini, à partir des Trente Glorieuses et de plus en plus depuis les années 1970, «la culture a été incorporée dans le système économique et politique des communications de masse » :

Le savoir-pouvoir des intellectuels en tant que couche sociale, filtré et sélectionné par des appareils technologiques et des systèmes productifs et institutionnels gigantesques, s'est liquéfié et fragmenté au sein de ces structures qui déterminent ou conditionnent les décisions fondamentales. Les intellectuels, inscrits dans des grands appareils de savoir-pouvoir obéissant à des centres de décision complexes et unifiés tant nationaux que multinationaux, n'ont plus aucune maîtrise de ces systèmes. Ils deviennent des simples travailleurs de la connaissance, obligés de se confronter à une instabilité perpétuelle, à la flexibilité et à la mobilité, et de développer ainsi une très grande capacité de recyclage professionnel. La culture humaniste-littéraire, émiettée en un ensemble incohérent d'informations et de connaissances, acquiert une valeur (très limitée) en tant que composante d'une formation pluridisciplinaire et flexible, capable de s'adapter à des conditions très variées (...). La ICT (Information and Communication Technology) a besoin de ce dispositif, puisque tant la production que la consommation des informations supposent une certaine élaboration du matériau informationnel. Mais il ne s'agit plus de la pratique traditionnelle de la médiation [des valeurs sociales par des intellectuels] - la médiation, qui tend à coïncider avec la simple imposition d'un produit marchand, est directement opérée par les appareils ${ }^{11}$.

Cette situation redistribue les hiérarchies et les rôles sociaux des intellectuels au sein du travail intellectuel :

La figure de l'intellectuel qui intervient dans la société grâce à l'autorité et au prestige qu'il tire de l'autonomie de son domaine et de l'indépendance culturelle et morale qu'elle garantit devient un vestige du passé. La partie « haute » du monde 
de la culture ne contrôle plus les processus de savoir-pouvoir dans lesquels elle est inscrite et qui déterminent la construction de l'opinion publique. Les intellectuels ne constituent plus l'unité idéologique d'une communauté ${ }^{12}$. radicale :

La marginalité sociale et la flexibilité de la production développent un Lumpenproletariat intellectuel. Le système semble demander des compétences non spécialisées et des jeunes intellectuels qui acceptent des emplois provisoires et partiels en utilisant des connaissances éclectiques et non cohérentes. Ainsi, l'expérience de la précarité professionnelle et de la marginalité sociale concerne des secteurs de plus en plus vastes du travail intellectuel, notamment parmi les jeunes générations ${ }^{13}$.

L'inutilité du travail intellectuel ne relève plus uniquement ni même principalement de la disjonction entre, d'une part, la culture et les savoirs « authentiques », et, de l'autre, les skills qui qualifient des tâches et des rôles socialement calculables, mais aussi de l'incapacité de ces skills de fournir un accès raisonnable à des positions sociales dominantes ou simplement stables et reconnues:

Les travailleurs de la connaissance, qui jouent un rôle crucial dans le fonctionnement des grands appareils technologiques et bureaucratiques et des institutions publiques, sont de moins en moins reconnus et valorisés socialement. Ils contribuent à la production sociale du sens et à l'élaboration des valeurs, mais ils sont inscrits dans des dispositifs qui désintègrent et broient les fonctions intellectuelles en leur soustrayant tout pouvoir réel et toute visibilité collective ${ }^{14}$.

\section{VII}

Quelles conséquences est-il possible de tirer de ces constats? Dans quelle mesure cette situation permet-elle de reconnaître des pratiques, des comportements ou des perspectives susceptibles d'incarner une contre-tendance face à la logique des rapports sociaux dominants?

Dans le texte que nous avons cité, Sergio Bologna se concentre sur le rapport entre le travail intellectuel et le marché capitaliste des compétences: «S'il faut traiter de la condition socio-économique du travail culturel aujourd'hui, il faut que notre discours porte sur la production et l'échange des compétences ; le travail de connaissance doit être provisoirement mis de côté $\aleph^{15}$. Le problème devient celui de l'amélioration de cette condition socio-économique et la suite de l'analyse de Sergio Bologna porte sur le statut et les revendications du travail indépendant ou freelance qui fait l'objet de l'activité associative et des mouvements des travailleurs du « tertiaire avancé $»^{16}$.

Mais il est peut-être possible de dire encore quelque chose à propos de la partie « incalculable » du travail intellectuel, et de son potentiel émancipateur situé en-deçà, ou ailleurs, par rapport à des pratiques organisées, associatives et quasi- ou post-syndicales.

Que faire avec cette composante incalculable du travail intellectuel? Comment développer les virtualités libératrices de son rapport singulier aux fins et à la perspective d'une activité non-aliénée? Ces questions sont distinctes de celle qui porte sur la légitimation et le statut du travail intellectuel par rapport au marché capitaliste des compétences : elles font allusion à ce que ce travail peut faire d'irréductible à un tel marché. 
Ainsi, Hans-Magnus Enzensberger a proposé de valoriser l'aspect d'«inutilité » qui caractérise la multiplicité foisonnante de savoirs, connaissances, langages et formes que chacun d'entre nous produit et transforme par-delà les impératifs des relations marchandes :

Comme l'indique son nom latin, Homo sapiens se bâtit une expérience à coups d'essais. Il ne le fait pas en tant que membre de l'espèce (...) mais pour son propre compte, à ses risques et périls. Il ne peut s'empêcher d'apprendre (...). Cette capacité n'a pas d'objectif déterminé, pas d'intérêt bien défini ${ }^{17}$.

30 Selon Enzensberger, cette capacité d'apprendre et de savoir fait du genre humain « une collectivité d'experts aux compétences personnelles déchaînées » se consacrant à des activités « parfaitement gratuites, pour lesquelles ne comptent ni le temps ni l'argent ${ }^{18}$, lesquelles ne jaillissent que de l'investissement subjectif passionné de chacun. Ces activités «inutiles» du point de vue capitaliste font partie d'un foisonnement de pratiques hétérogènes à la logique économique qui constituent, pour Enzensberger, la grande masse des comportements humains :

Les gens mènent, en effet, la plupart de leurs transactions en dehors des circuits de l'argent et du crédit (...). Souvent, ils travaillent gratis pro deo (...) et au grand désespoir des théoriciens [de l'économie néo-libérale] ne cessent de se comporter de la sorte ${ }^{19}$.

31 Il existe donc une vaste masse de comportements « improductifs » qui témoignent de la capacité humaine à se consacrer à des activités réglées, formalisées, demandant un effort, un engagement personnel et une discipline, mais que ne détermine aucun critère externe à l'expérience jubilatoire de leur exercice et de l'augmentation de notre puissance d'agir que celui-ci implique. Ces comportements constituent un moment fondamental des processus élémentaires de l'anthropogenèse. Leur persistance soutient le travail patient et invisible de l'humanisation de l'espèce, et représentent la tendance opposée au processus capitaliste qui livre des masses innombrables à une inutilité stérile et passive.

La composante «incalculable» du travail intellectuel relève de ces activités, dans la mesure où son seul but consiste dans l'enrichissement des pouvoirs de notre esprit que détermine le maniement des formes et des savoirs.

\section{VIII}

Fredric Jameson a rappelé que cette immanence du but à l'activité est centrale dans la vision de la «production » chez Bertolt Brecht, laquelle a directement partie liée avec sa vision du travail intellectuel :

L'activité elle-même est l'un des caractères de la connaissance et de l'art dans la mesure où ils appartiennent à la sphère de l'«utile»(...): l'utile devient ainsi imperceptiblement une fin à part entière (...) - une coïncidence hégélienne entre moyens et fins qui fait que l'activité devient digne d'être pratiquée par elle-même ${ }^{20}$.

Autrement dit, l'«utile» brechtien est précisément l'«inutile » du point de vue capitaliste, la vraie « utilité » réalisant la coïncidence entre moyens et fins dans l'usage intensif des gestes et des matières - un usage dont la seule fin est l'expression multilatérale de toutes les virtualités immanentes à ces gestes et à ces matières, aux sujets et aux objets de l'activité.

Ainsi, cette vision de l'activité rejoint celle d'Enzensberger et confirme que le travail intellectuel, vu dans ce qu'il a d'incalculable et de subjectif, peut constituer une figure de 
la libération générale de l'activité humaine. Mais cette capacité à représenter un horizon général d'émancipation ne relève pas que de l'immanence des fins à l'agir : elle a aussi partie liée avec la nature immédiatement collective du moment « incalculable » du travail intellectuel.

\section{Comme le rappelle Sergio Bologna :}

Le travail de connaissance (...) produit des idées sur lesquelles construire une mentalité, une capacité critique, une manière de vivre et de penser, une vision de la vie, un éthique et une politique. Le travail de connaissance produit des valeurs transmissibles, reproductibles, des idées qui peuvent être utiles aux autres. Dans un tel travail il y a toujours une tendance, une impulsion, sociales, le mouvement du don $^{21}$.

Cette précision permet d'éviter le risque d'une apologie trop immédiate, d'une confiance trop grande, à l'égard des vertus libératrices des activités inutiles, tel qu'on les trouve sans doute chez Enzensberger. Car les activités exercées par elles-mêmes peuvent incarner des idiosyncrasies parfaitement insignifiantes, que le capital ne «compte » pas en tant que telles, mais qui n'incarnent pas pour autant des tendances s'opposant à celle de la valorisation. Pour devenir effectivement libératrice, une activité ne doit pas simplement être inutile pour le capital : elle doit être utile pour l'avènement de rapports entre les hommes libérés du capital. L'immanence de la fin à l'agir ne suffit pas : pour que cet agir incarne réellement une contre-tendance, un comportement antagoniste, vis-à-vis de la valorisation capitaliste des pratiques et des conduites, il doit pouvoir être partagé et soutenir une augmentation commune des capacités humaines :

[Cette vision brechtienne de l'activité] a ses précurseurs: par-delà Marx nous pouvons reconnaître Goethe (...), le Goethe lecteur de Spinoza qui déclarait : «Je déteste tout ce qui n'augmente pas mon activité intellectuelle». "Intellectuel» tend insensiblement à coïncider avec «collectif», et l'activité acquiert ainsi une dimension historique ${ }^{22}$.

Le travail artistique de Brecht, l'« expérience du théâtre en tant qu'expérimentation collective », est «la promesse et l'exemple d'une coopération utopique $»^{23}$ : une activité intellectuelle qui est sa propre fin immanente mais dont l'organisation consciente et explicite ré-inscrit aussi autre chose- une expérience du collectif libéré que, dans certaines conjonctures, la politique a pu penser et réaliser sous d'autres formes et avec d'autres moyens :

Dans les années 1960, de nombreuses personnes comprirent que ce qui surgit dans une expérience collective réellement révolutionnaire n'est pas une foule anonyme mais un nouveau plan de l'être que Deleuze a appelé, d'après Eisenstein, le Dividu ${ }^{24}$.

Cette expérience, rappelle Jameson, a été oubliée et « ses traces ont été systématiquement effacées par le retour de toutes sortes d'individualismes désespérés » ${ }^{25}$.

\section{IX}

Serait-il possible que des activités intellectuelles s'organisent de telle sorte que cette expérience soit ré-inscrite dans leur exercice autonome, immanent et « inutile»? Ainsi, leur hétérogénéité par rapport aux relations capitalistes ne serait plus une détermination uniquement négative, et deviendrait la figure ${ }^{26}$ d'une autre organisation des rapports entre les êtres et les existences. Elles ne deviendraient pas pour autant des pratiques politiques, mais elles garderaient dans leur structure interne, dans leurs fins et moyens 
spécifiques, le souci d'une politique peut-être absente, et qu'elles pourraient, un jour, rencontrer.

Une méditation d'Antoine Vitez sur le théâtre exprime toute la contingence et la fragilité de cette rencontre entre les œuvres de l'« esprit » et la politique :

Or, justement, le théâtre, parce qu'il parle - simplement parce qu'il parle - fait figure d'agitateur, on croit qu'il fait promptement effet sur le monde, et je vois plutôt en lui un monastère en oraison (...). Le peuple est heureux de savoir qu'il est là, vivant en son sein, il n'a avec lui que des relations épisodiques et fragmentaires, ou imparfaites, jamais entièrement satisfaisantes. Et c'est toute l'action du théâtre : on sait qu'il travaille ${ }^{27}$.

41 La consistance de ce travail des formes et des signes, dont le théâtre est ici l'allégorie, n'est pas une tâche indigne : elle est ce sur quoi il ne faut pas céder aujourd'hui, pour les raisons que nous avons essayé d'indiquer.

\section{$X$}

Mais il ne faudra pas oublier que tous les termes actuels de la problématique du travail intellectuel deviendraient radicalement insuffisants si la rencontre avec un processus politique consistant soumettait cette problématique et ce travail à l'épreuve d'un réel dont le nom a été pendant deux siècles celui de "révolution». Car dans un processus "révolutionnaire» le problème du bon usage des contradictions internes au travail intellectuel tel qu'il existe avant la "révolution » tend à être remplacé par celui du déplacement des fonctions intellectuelles et de leurs porteurs. Nulle «révolution » ne saurait éviter de devenir elle-même le lieu d'un partage où prennent forme des idées, « une capacité critique, une manière de vivre et de penser, une vision de la vie, un éthique et une politique ", par-delà les rôles et les fonctions actuelles.

Ainsi, ce qu'on peut faire avec et dans le travail intellectuel aujourd'hui, les moments et les gestes de libération qu'il est possible d'arracher tant à son inutilité qu'à sa subsomption par le capital et l'ordre social dominant, attendent toujours une rencontre qui accomplira et abolira les contradictions dans lesquelles nous aurons vécu:

[Il s'agit d'] élaborer des modèles d'écriture critique, de langage d'essayistes, d'information écrite, d'organisation d'enquêtes et d'études, de traduction, de direction dans le domaine des disciplines littéraires; des modèles qui ne se pensent pas comme des concurrents pour les modèles existants, car ils savent que la réalité révolutionnaire, lorsqu'elle survient, ne peut éviter de rendre méconnaissables tous les modèles, y compris les plus généreux ${ }^{28}$.

\section{NOTES}

1. Luciano Ferrari Bravo, «Lavoro e politica » (1996), in Dal fordismo alla globalizzazione, a cura di S. Bologna, Rome, Manifestolibri, 2001, p. 233-234.

2. Sergio Bologna, «Conoscenza, cultura, competenza. Come difendere il valore del lavoro intellettuale e creativo: un contributo alla discussione», intervention d'ouverture de 
l'Assemblée sur le travail culturel, dans le cadre de la manifestation Libri come, Rome, 11 mars 2012 (http://www.uninomade.org/wp/wp-content/uploads/2012/03/11_marzo4-1.pdf).

3. Ibid.

4. Jean-Claude Milner, De l'école, Paris, Seuil, 1984, p. 122.

5. J.-Cl. Milner, De l'école, op. cit., p. 116-117.

6. Paul Lafargue, Le socialisme et les intellectuels, Paris, Giard \& Brière, 1900, p. 35-36.

7. Alain Badiou, Notre mal vient de plus loin, Paris, Fayard, 2016, p. 33-34.

8. Pierre-Noël Giraud, L'homme inutile, Paris, Odile Jacob, 2015, p. 12-13.

9. Slavoj Žižek, Pour défendre les causes perdues, Paris, Flammarion, 2008, p. 329-330.

10. Jean-Paul Sartre, Plaidoyer pour les intellectuels, Paris, Gallimard, 1972, p. 13.

11. Romano Luperini, Tramonto e resistenza della critica, Macerata, Quodlibet, 2013, p. 16-17. Cf. les remarques de J.-Cl. Milner: "Aujourd'hui, l'intellectuel est convié à subir le même sort que l'artisan ou l'ouvrier spécialisé : renoncer à ce qui fait son être, pour devenir le pendant, dans l'ordre du travail intellectuel, du travailleur sans qualification, du travailleur abstrait, propre à toutes les tâches requises par la production. Le manœuvre intellectuel, voilà la forme qui se dessine à l'horizon. Tout comme le manœuvre manuel, le manœuvre intellectuel est propre à tout puisqu'il n'est propre à rien.il n'a pas à être payé beaucoup puisqu'il n'a aucune qualification. Il est aisément remplaçable puisque tous les manœuvres se valent. La figure moderne qui le réalise dans la société en général, est le polyvalent intellectuel : le plumitif des journaux, le présentateur moyen de la radio et de la télévision, c'est enfin l'instituteur modèle de la Corporation » (J.-Cl. Milner, De l'école, op. cit., p. 126).

12. Ibid., p. 17.

13. Ibid.

14. Ibid., p. 18.

15. S. Bologna, «Conoscenza, cultura, competenza. Come difendere il valore del lavoro intellettuale e creativo : un contributo alla discussione », op. cit.

16. Cf. les activité de l'association ACTA: http://www.actainrete.it/wp-content/ uploads/2014/05/Manifesto-dei-lavoratori-autonomi-di-seconda-generazione.pdf.

17. Hans-Magnus Enzensberger, Le panoptique, Paris, Alma, 2014, p. 57.

18. Ibid., p. 59.

19. Ibid., p. 17-18.

20. Fredric Jameson, Brecht and Method, Londres, Verso, 1998, p. 6.

21. S. Bologna, «Conoscenza, cultura, competenza. Come difendere il valore del lavoro intellettuale e creativo : un contributo alla discussione », op. cit.

22. F. Jameson, Brecht and Method, op. cit., p. 226.

23. Ibid., p. 15.

24. Ibid., p. 14-15.

25. Ibid., p. 15.

26. Sur la notion de figure, voir l'article de Gabriele Fichera, «Ruines que nous devrons reconnaitre. Le dernier Fortini et la Figura », in Cahiers du GRM, 7, 2015, https:// grm.revues.org/666.

27. Antoine Vitez, le 25 décembre 1977, cité in François Regnault, Solstices. Ecrits sur le théâtre 2, Paris, Actes Sud, 2002, p. 56.

28. Franco Fortini, «Verifica dei poteri » (1964), in Saggi ed epigrammi, a cura di Luca Lenzini, Milan, Mondadori, 2003, p. 174. 


\section{RÉSUMÉS}

L'article reprend la question du travail intellectuel à la lumière de la vision opéraïste du « travail vivant ».

INDEX

Index chronologique : XXe siècle

Thèmes : marxisme, sociologie des intellectuels

Index géographique : Europe

Mots-clés : travail intellectuel, travail vivant, homme inutile, Ferrari Bravo Luciano,

Enzensberger Hans-Magnus, Vitez Antoine, Brecht Bertolt, Jameson Fredric

\section{AUTEUR}

\section{ANDREA CAVAZZINI}

Andrea Cavazzini est vice-président de l'Association Groupe de Recherches Matérialistes. 